\title{
A Study on Performance Enhancement of Parabolic Trough Collector
}

\author{
Aditya Bawane $^{{ }^{*}}$, Sanjay Lakade ${ }^{\text {, }}$, and Virendra Bhojwani ${ }^{2}$ \\ ${ }^{1}$ Pimpri Chinchwad College of Engineering, Pune, India \\ ${ }^{2}$ MIT-ADT University, Pune, India.
}

\begin{abstract}
Solar energy is available in abundant quantity which can be utilized for thermal and power generation applications. The maximum solar energy extraction for application is challenging. This review focuses on the performance enhancement of parabolic trough collector. Heat transfer through absorber tube, various nanofluids with concentration is stated. Thermal efficiency increases due to the use of parabolic collector with booster reflector and glass cover over the system performance. The complex 3D analysis (ANSYS) gives accurate distribution of heat flux over the absorber tube.
\end{abstract}

Keywords: Parabolic trough collector, secondary booster reflector.

\section{Introduction}

Many technologies have been developed to make use of solar radiation to reduce the dependency on the exhaustable energy sources. Sun releases energy in the form of radiation which is $1.74 \times 10^{\wedge} 7 \mathrm{~W}$ at the upper layer of the earth. $51 \%$ of these energy reaches to earth surface which is huge [1]. Solar energy is free, but capturing, processing and storaging costs are high. There are many methods to utilize the solar energy in low to high temperature range. Among these different solar collector are flat plate collector, evacuated tube collector, parabolic trough collector (PTC), compound collector, linear Fresnel reflector, parabolic dish collector, heliostat field collector.

The flat plate collectors are used for applications such as domestic water heating for low temperature (120$\left.140^{\circ} \mathrm{C}\right)$.PTC and $\mathrm{CPC}$ are used to steam generation plant. PTC has parabolic shaped mirror, absorber and receiver tube, and tracking mechanism. It can reach the temperature range of $350-400{ }^{\circ} \mathrm{C}$. CPC can be used for low and medium temperatures of $60-240{ }^{\circ} \mathrm{C}$ [2] with high efficiency and low heat loss. Linear Fresnel reflector have long rows of mirror which direct the solar radiation to the downward facing fixed receiver.

\section{Parabolic Trough Collector}

In PTC, Solar energy incident on parabolic shaped mirror then it reflected to the receiver tube through glass covered absorber tube. The fluid that circulates through the receiver tube is heated and used for additional applications such as steam production. PTC uses single axis or two axis tracking system. Mostly two axes system are arranged in north-south direction for tracking the sun and it is moved along east-west direction for high optical efficiency. Single axis tracking system with receiver tube is arranged in east-west direction, which results in lesser efficiency as compared to dual axes tracking. PTC with have $60-70 \%$ thermal efficiency with evacuated receiver tube which is $10 \%$ higher than non-evacuated tube.

\subsection{Thermal Analysis of PTC Receiver}

The heat transfer through receiver tube occurs through three different heat transmission modes - conduction, convection and radiation.

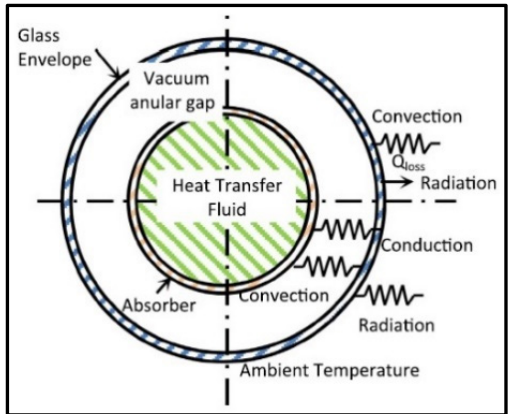

Fig. 1. Cross-sectional receiver for the heat transmission The transfer of convection heat take place whenever medium changes and it greatly depends upon geometry, surface roughness and fluid properties [3]. Heat transmission by conduction through glass and receiver tube is related by Fourier law: [4]

$q_{\text {pipe (in-out) }}=\frac{2 \pi K_{c}}{\ln \left(\frac{D_{\text {pipe, in }}}{D_{\text {pipe,out }}}\right)}\left(T_{\text {in }}-T_{\text {out }}\right)$

Where $K_{c}$ is the thermal conductivity of receiver $(\mathrm{W} / \mathrm{m}$ $\left.{ }^{\circ} \mathrm{C}\right)$.

Heat transfer by convection between the fluid transfering heat and inside surface of receiver tube can be expressed as ;

*Corresponding author: aditya23892@gmail.com 
$q_{f, \text { conv }}=\pi D_{p i} h_{f}\left(T_{p i}-T_{\text {fluid }}\right)$

Where $h_{f}$ is the coefficient of convection heat transfer at the internal diameter of tube $\left(\mathrm{W} / \mathrm{m}{ }^{\circ} \mathrm{C}\right) ; D_{p i}$ is the diameter (internal) of the receiver $(\mathrm{m}) ; T_{p i}$ is internal surface temperature of tube $\left({ }^{\circ} \mathrm{C}\right)$ and $T_{\text {fluid }}$ is the average temperature of the circulating fluid $\left({ }^{\circ} \mathrm{C}\right)$.

Radiation does not require any heat transfer medium, it can be transmitted, absorbed or reflected. The heat transfer due to radiation between the envelope of glass and the receiver pipe considering diffused reflection and radiation is given by equation (3)

$$
q_{p i-p o, r a d}=\frac{\sigma \pi D_{p i, o}\left(T_{p o}^{4}-T_{g i}^{4}\right)}{\frac{1}{\varepsilon_{p o}}+\left(\frac{\left(1-\varepsilon_{g i}\right) D_{p o}}{\varepsilon_{g i} D_{g i}}\right)}
$$

\section{Heat Transfer Enhanancement}

\subsection{Nanofluids}

The increased heat transfer for the base substance with insert in the laminar region was increased $20-300 \%$ as compared to regular receiver [5]. This is due to the turbulence enhances the diffusion of energy and hence the heat transfer. $\mathrm{TiO}_{2} / \mathrm{De}$ - ionized water with nano fluid increases the output of PTC. $\mathrm{TiO}_{2}$ will achieve an improvement in maximum efficiency of $8.66 \%$ (57\%) higher than the solution of water base at a volume concentration of $0.2 \%$ having mass flow of $0.0667 \mathrm{~kg} / \mathrm{s}$ [6]. These nanofluids have an energy factor of $9.51 \%$ higher than water. Distilled and deionized water and alumina nanoparticles $\left(\mathrm{Al}_{2} \mathrm{O}_{3}\right)$ were used in volume fraction of $1 \%$ and $3 \%$. The addition of nanoparticles increases the thermal properties of the fluid, thereby allowing energy exchange between the working fluid and the tube to be increased. For 3\% volume fraction of nanofluid, the maximal efficiency was $52.4 \%$ and $40.8 \%$ for water for various ambient conditions and incident angles. Nano fluid had a maximum efficiency of $57.7 \%$ for nanofluid and $46.5 \%$ for water for $1 \%$ volume fraction of nanofluid. The maximum efficiency was found for the small angle of incident [7]. For a small solar radiation value, the outlet nanofluid temperature was higher than water.

\subsubsection{Different Geometry for Receiver Tube}

The absorber tube of PTC was connected with twisted tubes which resulted in enhancement of energy exchange. Five reviver tubes were used for receiver tube each one with overlapped flows of two types. The first one is the flow-through twisted inner tubes of different hydraulic diameters (single, double and triple). Second with reverse flow via an external tube. The measurements showed a significant improvement in the energy exchange of the single twisted tube (STT) and a greater increase in overlapped the triple twisted tube (TTT) for the single receiver tube. This is because the pressure difference for TTT is greater than STT [8].

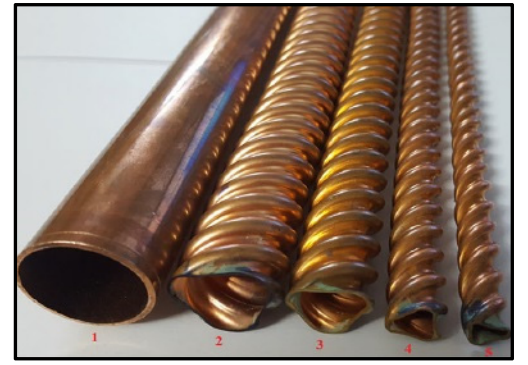

Fig. 2. Twisted tubes [8]

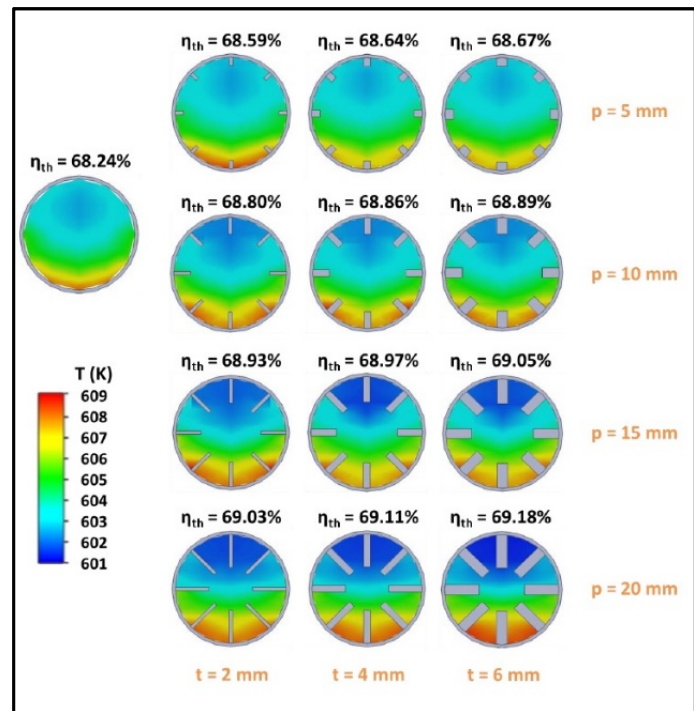

Fig. 3. Thermal oil distribution for all tested absorbers at $600 \mathrm{~K}$ and $150 \mathrm{~L} / \mathrm{min}$ [9].

Twelve absorber tubes are compared with smooth tube with internally finned length of $5 \mathrm{~mm}$ to $20 \mathrm{~mm}$ and a thickness of 2 to $6 \mathrm{~mm}$. As the number of fin increases the efficiency will increase but losses occurs at high pressure. The fin's length is significantly higher than its thickness [9]. However thickness have significant effect on pressure losses.

\subsection{Receiver and Optical Efficiency}

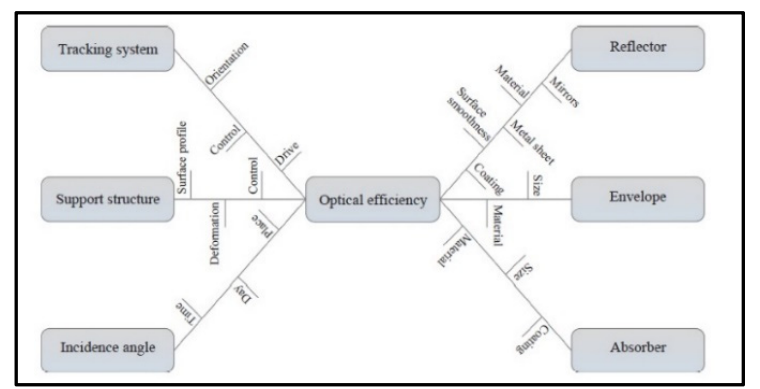

Fig. 4. Parameters affecting the optical efficiency [16].

Silver and Aluminium mirrors are most commonly used materials for mirrors having ideal reflectance of 0.99 [16]. Fig. 5. Shows the parameter affecting the optical efficiency. The unit length cost for receiver decreases when size of solar field expands from 2.8 hectares to 10 hectares (almost halved about $\$ 1400$ to $\$ 760$ ). Efficiency depends on reflective area, aperture area and solar field area. For large capacity plants it is possible to cut down the cost in each sector. The labour cost for mechanical 
work can be drops by $48 \%$ for 10 hectares and $75 \%$ for 160 hectares. Where as civil cost remain same. Copper can be used as receiver tube material for PTC considering thermal stresses and bending phenomenon [10]. Optical performance reduces with respect to time and glass breaks. To prevent this risk, the thickness of metal ring should be minimal enough and coefficient of thermal expansion between the glass cover and the metal ring should be lower than 7\% [11]. The best method to eliminate the conduction losses from receiver tube is creating vacuum between glass cover and receiver tube. Borosilicate glass and stainless steel are good material for vacuum. Getter is necessary to maintain vacuum [10]. Data from existing PTC plant shown $55 \%$ of failure due to broken glass and $29 \%$ due to vacuum loss. The receiver cost is $30 \%$ of plant cost having payback period of $1-5$ years. The bent receiver tube may touch glass cover and damage it [12]. In order to concentrate thermal photovoltaic collectors, increase in inlet temperature increases exergy efficiency but reduces thermal and electrical efficiencies [13].

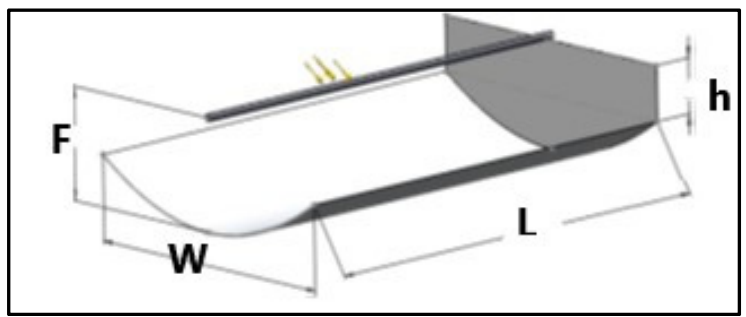

Fig. 5. PTC with booster reflector [14]

A secondary vertical reflector is placed at the edge of collector to enhance the optical performance. This reduces the losses at the end of receiver of PTC by reflecting back to receiver. With higher solar incident angle, the optical enhancement with booster reflector is higher. With a ratio of 0.236 (focal), the annual enhancement is $21.7 \%$. In winter it is greater and smaller in summer as a percentage. When the system operates at high temperatures, the thermal improvement is higher [14]. The flat glass coverup over the PTC increases the thermal output by $9 \%$ [15]. This is due to the air trapped inside the envolope heat up the inside air and reduces the convection losses.

\section{CFD Analysis}

To predict thermal performance, it is useful to analyze PTC by $1 \mathrm{D}$ and less complex 2D. The complex 3D analysis is most suitable and accurate and it provides all information about actual distribution of heat flux on the receiver, which cannot be achieved by $1 \mathrm{D}$ or $2 \mathrm{D}$ model.

Fig.7. a) and b) shows the distribution of temperature and heat flux on receiver pipe. For selection of optimum receiver tube properties $3 \mathrm{D}$ analysis is useful for prediction of minimum and maximum temperature. For this purpose CFD analysis is useful for creating and meshing the geometry, defining the boundary conditions and heat transfer of fluid flow. MTRC method used to provide an non homogenous solar flux distribution over the heat collecting element for optical analysis [17].

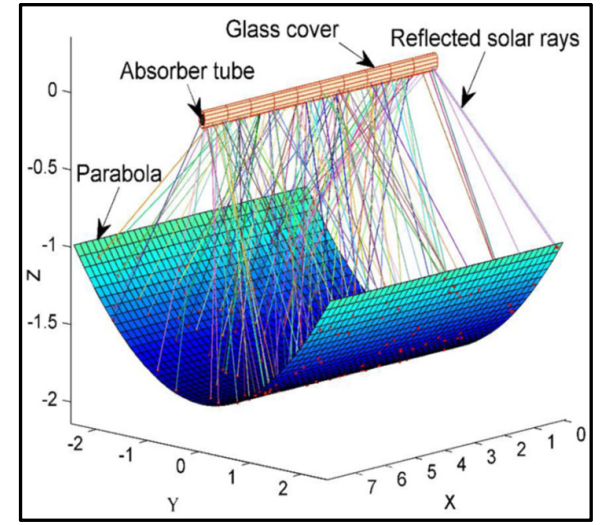

Fig. 6. Solar concentration mechanism obtained using 3D analysis in parabolic trough collector [17]

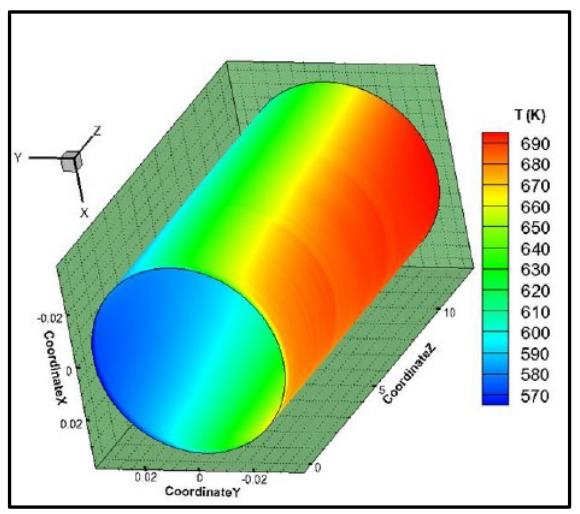

Fig. 7. a) External temperature distribution [17]

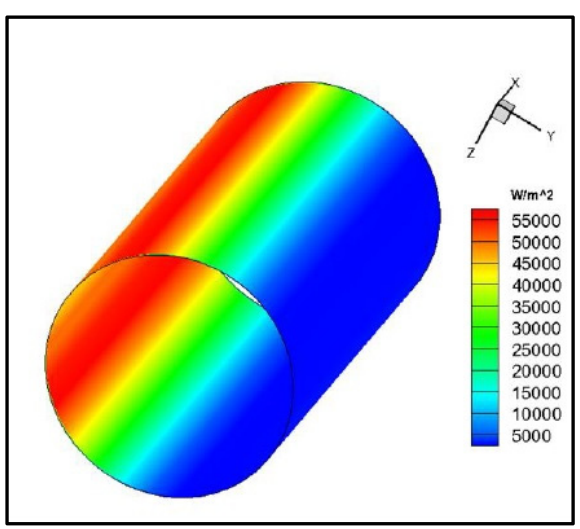

Fig. 7. b) External heat flux distribution [17]

\section{Conclusion}

This study focuses on mathematical modeling and techniques used to improve the energy exchange in PTC. The following conclusion are drawn from review:

a) In laminar flow regimes with inserts, the heat transfer improvement is significant. Nanofluids can increase the energy factor to $9 \%$ as compared to water.

b) Increase in fin numbers in receiver tube increases the efficiency but it results into higher pressure losses. Fin thickness have significant effect on pressure losses. 
c) PTC with a secondary booster reflector raises the annual enhancement by $21.7 \%$ with a focal ratio of 0.236 .

d) Glass cover over the PTC increases the thermal efficiency by $9 \%$.

e) The complex 3D model is most accurate for actual heat flux distribution over a receiver tube.

\section{References}

1. W. Fuqiang, C. Ziming, T. Jianyua, Y. Yuan, S. Yong, L. Linhua, Progress in concentrated solar power technology with parabolic trough collector system: A comprehensive review, Renewable and Sustainable Energy Reviews 79 (2017)

2. V. Pranesh, R. Velraj, S. Christopher, V. Kumaresan, A 50 year review of basic and applied research in compound parabolic concentrating solar thermal collector for domestic and industrial applications, Solar Energy 187 (2019).

3. A. Abdulhameda, N. Adam, M. Ab-Kadir, A. Hairuddin, Review of solar parabolic-trough collector geometrical and thermal analyses, performance, and applications, Renewable and Sustainable Energy Reviews 91 (2018)

4. L. S. Conrado, A. R. Pulido, G. Calderon, Thermal performance of parabolic trough solar collectors, Renewable and Sustainable Energy Reviews 67 (2017)

5. H. M. Sanse, Renewable and Sustainable Energy Reviews (to be published)

6. J. Subramani, P.K. Nagarajan, Omid Mahian, Ravishankar Sathyamurthy, Efficiency and heat transfer improvements in a parabolic trough solar collector using $\mathrm{TiO}_{2}$ nanofluids under turbulent flow regime, Renewable Energy (2017)

7. B. Rios MS, Rivera-Solorio, G. Cuéllar AJ, Thermal performance of a parabolic trough linear collector using $\mathrm{Al}_{2} \mathrm{O}_{3} / \mathrm{H}_{2} \mathrm{O}$ nanofluids, Renewable Energy (2018)

8. Ra'ad K. Mohammed Aldulaimi, An Innovative Receiver Design for a Parabolic Trough Solar Collector Using Overlapped and Reverse Flow: An Experimental Study, Arabian Journal for Science and Engineering, (2019).

9. E. Bellos, C. Tzivanidis, D. Tsimpoukis, Thermal enhancement of parabolic trough collector with internally finned absorbers, Solar Energy · (2017)

10. S. Akbarzadeh, M. Sadegh Valipour, Heat transfer enhancement in parabolic trough collectors: A comprehensive review, Renewable and Sustainable Energy Reviews 92 (2018)

11. Ze-Dong Cheng, Xue-Ru Zhao, Ya-Ling He, Novel optical efficiency formulas for parabolic trough solar collectors: Computing method and applications, Applied Energy 224 (2018)

12. Mohammed Soudani, Kamal E. Aiadi, D. Bechki and S. Chihi, Experimental and theoretical study of Parabolic trough collector (PTC) with a flat glass cover in the region of algerian sahara (Ouargla), Journal of Mechanical Science and Technology (2017)
13. M. Valizadeh, F. Sarhaddi, M. Adeli, Exergy performance assessment of a linear parabolic trough photovoltaic thermal collector, Renewable Energy 138 (2019)

14. Evangelos Bellos, C. Tzivanidis, Investigation of a booster secondary reflector for a parabolic trough solar Collector, Solar Energy 179 (2019)

15. M. E. Soudani, K. E. Aiadi, D. Bechki and S. Chihi, Experimental and theoretical study of Parabolic trough collector (PTC) with a flat glass cover in the region of algerian sahara (Ouargla), Journal of Mechanical Science and Technology (2017)

16. Mokheimer EM, Dabwan YN, Habib MA, Said SA, Al-Sulaiman FA. Techno-economic performance analysis of parabolic trough collector in Dhahran, Saudi Arabia. Energy Convers Manage (2014)

17. B. Agagna, A. Smaili, Q. Falcoz, O. Behar, Experimental and numerical study of parabolic trough solar collector of MicroSol-R tests platform, Experimental Thermal and Fluid Science (2018). 